\title{
ÉTICA EN EL CURRÍCULO DE LAS CARRERAS DE ODONTOLOGÍA
}

\author{
Carlos Zaror Sánchez y Carolina Vergara González ${ }^{* *}$
}

Resumen: Se realizó una revisión bibliográfica sobre la incorporación de la ética en el currículo de la carrera de odontología. La información recopilada se sistematizó para configurar un planteamiento general en torno a este tema, poniendo énfasis en su importancia dentro del currículo, así como en los objetivos, contenidos y estrategias involucrados en la enseñanza de esta disciplina.

Palabras clave: ética, bioética, bioética en odontología

\section{ETHICS IN THE CURRICULUM OF ODONTOLOGICAL CAREERS}

Abstract: A bibliographical review was performed related to the incorporation of ethics in the curriculum of an odontology career. The information compiled was processed in order to create a general suggestion around this theme, placing emphasis on its importance inside the curriculum, as well as in the objectives, content, and strategies involved in the instruction of this discipline.

Key words: ethics, bioethics, bioethics in odontology

\section{ÉTICA NO CURRÍCULO DOS CURSOS DE ODONTOLOGIA}

Resumo: Realizou-se uma revisão bibliográfica sobre a incorporação da ética no currículo do curso de odontologia. A informação recopilada foi sistematizada para configurar um questionamento geral em torno deste tema, pondo-se ênfase em sua importância dentro do currículo, assim como nos objetivos, conteúdos e estratégias envolvidos no ensino desta disciplina.

Palavras-chave: ética, bioética, bioética em odontologia

* Cirujano Dentista. Departamento de Odontología Integral, Facultad de Medicina, Universidad de La Frontera, Chile

** Cirujano Dentista. Servicio de Salud Araucanía Sur, Chile

Correspondencia: cezarors@gmail.com 


\section{Introducción}

Como en otras disciplinas del área de la salud, la ética forma parte de la práctica diaria en la profesión odontológica. Por esta razón, resulta sorprendente que todavía en la mayoría de los currículos de universidades de todo el mundo se destina muy poco tiempo a la formación y al estudio de esta disciplina(1-5). Esto implica dejar a los profesionales sin preparación en la toma de decisiones éticas de tanta importancia dentro del contexto social actual.

Los cambios generacionales, la transformación de valores sociales a través de la historia, el avance de la ciencia y la tecnología, el mayor conocimiento por parte del paciente, entre otros factores, implican una serie de dilemas y conductas éticas que se deben entender y analizar. Es precisamente la universidad el lugar donde se adquieren y modifican los patrones o modelos de conducta que se aplicarán en la vida profesional.

Si bien la ética médica tiene 2500 años de historia formando parte de la educación en las ciencias de la salud, sólo en los últimos 30 ha sido incluida en términos formales en los currículos médicos(6). En un comienzo, esta disciplina fue enseñada en combinación con cursos de áreas afines como Historia Dental, Gestión, Jurisprudencia, Deontología, Medicina Legal, cursos basados en metodologías con poca o nula oportunidad de diálogo por parte de los estudiantes(5). Esta situación ha llevado a una escasa motivación por la ética en la educación dental y a un escaso conocimiento de estos temas por parte del futuro profesional $(6,7)$. Por otro lado, este tipo de metodología sólo permite tener conocimiento de la ética desde un punto de vista teórico, pero sin permitir cambios conductuales que lleven a una correcta toma de decisión(8).

En relación con lo anterior, Bertolami(9) detectó tres principales debilidades de la educación ética en los currículos odontológicos. Su publicación abrió un debate mundial en relación con el tema, pues encontró que la actual educación ética en odontología no reconoce que:

- La educación no es la respuesta a todo.

- La actual enseñanza de la ética es aburrida.

- Los contenidos de los cursos son cualitativamente inadecuados para llevar a cabo un verdadero cambio de conducta.

En la mayoría de los currículos odontológicos el ramo de ética es enseñado en forma tradicional mediante una clase expositiva. Ozar notó que hace tiempo existía la inquietud por realizar innovación de la enseñanza de la ética en odontología y se estaba buscando la forma de hacerla más práctica y entretenida(10-12).

Con respecto a esta inquietud, las nuevas tendencias en materia de educación de esta asignatura incluyen cursos separados de ética, dictados frecuentemente durante el primer ańo de estudio, reforzados en forma continua de manera horizontal y vertical a través de los diferentes niveles, siendo el refuerzo más importante el realizado durante los cursos en los cuales el alumno realiza su práctica clínica(13). La finalidad de estos cambios es que los dilemas éticos sean abordados en el momento en que se van presentando de forma natural. Un amplio consenso en la literatura científica disponible avala que la ética debe ser enseñada de forma multidisciplinaria e interprofesional $(11,12,14)$.

Con relación a lo anterior, y puesto que la disciplina tiene un carácter multifactorial, los nuevos cursos de ética demandan una formación en las distintas áreas de la ciencia y humanidades, tales como filosofía, antropología, sociología, medicina, teología, derecho; por lo tanto, requiere del intercambio de ideas con los representantes de las diferentes áreas participantes $(2,15)$.

A continuación se expondrá, de acuerdo con la literatura consultada, cómo se está enseñando la ética dentro del currículo odontológico y cuáles son los cambios que se están produciendo en esta materia.

\section{¿Por qué incorporar ética en el currículo odontológico?}

Para decidir incorporar esta disciplina al currículo de odontología, lo primero es comprender qué beneficios tiene para nuestros estudiantes y cuál será la relevancia de su inclusión.

El desarrollo de habilidades técnicas y la adquisición de conocimientos científicos son dos elementos indispensables pero no suficientes para proporcionar un estado de salud oral de calidad a los pacientes. El factor más importante es el compromiso de los odontólogos para aplicar sus capacidades con integridad, teniendo como objetivo principal los intereses del paciente y su salud oral. La justificación para enseñar ética en odontología es facilitar el desarrollo profesional y personal de los estudiantes para que se conviertan en seres humanos responsables, tanto social como profesionalmente(16). 
Por otro lado, los continuos avances en los conocimientos y la tecnología en el área de las ciencias de la salud requieren que los profesionales sean capaces de enfrentar adecuadamente un problema ético, valorar sus implicancias, decidir el curso de acción e implementar los pasos necesarios para resolverlo. Estas habilidades deben ser enseñadas y entrenadas(17).

Esta formación es mucho más eficiente cuando se realiza paralelamente a la educación técnico-científica, es decir, en la universidad(18). Los primeros contactos con docentes, autoridades, compañeros y pacientes fomentan el desarrollo de conductas éticamente co$\operatorname{rrectas}(1)$.

La educación ética capacita al estudiante para reconocer conflictos éticos, participar en forma activa y significativa en comités de ética, respetar los límites éticos de la investigación, trabajar en equipos interdisciplinarios de manera racional y pluralista, entre otros. También permite al estudiante estar consciente de las responsabilidades que asume desde el momento en que decide integrarse a la profesión odontológica(19), en una sociedad cada vez más demandante, informada y conocedora de sus derechos.

\section{Enseñanza de la ética: aspectos sicológicos}

Desde el momento en que se asume la necesidad de incorporar la ética en el currículo de una carrera universitaria, es importante tener la convicción de que en esta etapa sí es posible lograr cambios o generar reflexión en torno a dilemas. Bebeau(20) identifica algunos mitos en torno al potencial para influir en el desarrollo ético de los profesionales. Uno de ellos sostiene que el carácter está determinado desde antes de la pubertad y que no se puede enseñar a alguien a ser ético después de eso. Otro argumenta que, incluso si se enseńa ética, no hay garantía de que la persona actuará éticamente. Una tercera idea sostiene que la gente sólo cambiará a través de la opresión.

La investigación sicológica indica que los individuos a veces fallan en hacer lo correcto por las siguientes razones:

- Un individuo puede estar ajeno a los asuntos éticos que se le presentan. Cuando los individuos se incorporan a un nuevo campo de acción, como una profesión, necesitan aprender a integrar la información técnica de la disciplina con lo que ellos entienden sobre el rol profesional y sus obli- gaciones, así como también entender la manera en la que un paciente podría comportarse, en el caso de la profesión odontológica. Si un profesional es insensible a las necesidades de los otros o si una situación es muy ambigua el profesional podría fallar en actuar moralmente.

- Una persona puede ser incapaz de formular un curso de acción moralmente defendible. La historia está repleta de situaciones que reflejan esta idea; por ejemplo, los oficiales nazis defendieron el asesinato de millones de judíos sobre la base de su obligación de obedecer la autoridad.

- Un individuo puede fallar en darle prioridad a asuntos morales. Por ejemplo, la profesión odontológica está preocupada por la gran cantidad de dentistas y la decreciente incidencia de la enfermedad, condiciones que podrían sobreponer intereses económicos a la decisión profesionalmente correcta.

- Las fallas morales pueden resultar de una incapacidad o falta de voluntad para implementar un plan de acción efectivo. La perseverancia, competencia y carácter son cualidades requeridas para lograrlo. Habilidades interpersonales deficientes y poca capacidad para resolver problemas interfieren con la efectiva resolución de un dilema.

Entonces, la incorporación de la ética en el currículum, según la visión de Rest(20), debería estar orientada a desarrollar en los estudiantes habilidades que les permitan desempeñarse como profesionales moralmente responsables y minimizar estas debilidades. Dos conceptos se vinculan con dichas habilidades: la "sensibilidad ética" -capacidad para identificar dilemas éticos en las situaciones que ellos confrontan-y la "opinión o juicio moral" -habilidad de formular una opinión moral bien argumentada. Ambos deben ser tomados en cuenta en la formulación de un programa de ética. Para cada uno de ellos se podrán emplear diversas estrategias, como se verá más adelante.

Ahora, ¿̨pueden estas estrategias, además de sensibilizar al futuro profesional en temas éticos, influir en su comportamiento? Rest, en 1986, recopiló una extensa cantidad de investigaciones del ámbito psicológico que contradicen la creencia de que los adultos mantienen una firme e inalterable escala de valores que rigen su conducta. Bebeau resume estos hallazgos y los complementa con investigaciones en estudiantes de odontología: 
- Se producen cambios importantes en los adultos jóvenes según las estrategias que se utilicen para enfrentar temas éticos. Estudios muestran que el desarrollo ético ocurre mayormente después de la adolescencia.

- Estas transformaciones se relacionan con las reconceptualizaciones acerca de cómo una persona entiende la sociedad y su función en ésta, interpretando y definiendo problemas morales con relación a la etapa de desarrollo en la que se encuentra, dando como resultado intuiciones diferentes de qué es lo correcto y justo.

- La vocación universitaria está poderosamente asociada con el desarrollo del juicio moral, el cual tiende a detenerse cuando la persona deja la educación. Las personas que tienen más desarrollado el juicio moral tienden a ser más reflexivas e interesadas en continuar su propio crecimiento y educación, teniendo al mismo tiempo la disposición para aprender más sobre temas sociales y participar en ellos.

- Los intentos de desarrollar la conciencia por problemas morales así como su razonamiento y juicio a través de la educación, son efectivos. Los programas educacionales ponen énfasis en la discusión de dilemas morales con los pares controversiales, lo que produce resultados satisfactorios, especialmente en estudiantes de 20 a 30 ańos. Por el contrario, cursos teóricos cortos parecen no ser muy efectivos.

- No hay diferencia significativa entre hombres y mujeres con relación a la sensibilidad ética y el juicio moral, sino que depende básicamente del nivel de educación.

- Hay estudios que relacionan percepción y juicio moral con el comportamiento del profesional en la vida real. Mientras que, ciertamente, no hay garantías de que los cursos de ética impartidos puedan asegurar un comportamiento ético, muchos estudios muestran que el juicio moral se relaciona significativamente con una amplia variedad de comportamientos y actitudes.

La educación es una experiencia de reflexión que guía el cambio de comportamiento. Esta debe tener como propósito disipar la idea de que la moralidad es opcional. Por el contrario, los estudiantes deben comprender que la ética es esencial para vivir en sociedad y desenvolverse como profesionales en el sentido más amplio de la palabra(16).

\section{Objetivos de la enseñanza de la ética en odontología}

Según Bridgman(19), el propósito final y más importante es "la formación de buenos dentistas quienes realcen y promuevan la salud general y buco dentaria, además de proporcionar bienestar a la gente que atienden de manera justa, respetando sus dignidad, autonomía y derechos".

Este deseo debe verse plasmado en objetivos claramente definidos, que orienten el desarrollo del curso y que permitan realizar una evaluación satisfactoria de los logros.

Los objetivos que se mencionan a continuación corresponden a un resumen de los encontrados en diversas publicaciones médicas y odontológicas $(16,19)$ :

- Sensibilizar al estudiante de odontología con la dimensión moral de la práctica profesional.

- Desarrollar habilidades para realizar análisis éticos.

- Fomentar el respeto por el desacuerdo y la tolerancia ante la ambigüedad.

- Ayudar en la explicación de las responsabilidades morales que se adquieren desde el momento en que se forma parte de la profesión odontológica.

- Motivar al estudiante a tener una formación continua en el campo de la ética profesional.

- Conocer las principales obligaciones profesionales de los dentistas y las instituciones que regulan la actividad.

- Apreciar el razonamiento ético como un componente natural e integral de la práctica clínica y del proceso de toma de decisiones.

- Comprender que casi todas las decisiones en la práctica clínica diaria tienen un componente ético, tanto como las situaciones más excepcionales de la odontología.

- Reconocer los límites éticos de la investigación científica.

\section{Contenidos del programa de ética para odontólogos}

Dejando de lado el debate acerca de la eficacia de la incorporación de la ética en el currículo de odontología, tampoco existe total consenso con relación a los contenidos que deberían ser incluidos en el programa. 
Los temas éticos en las carreras del área de la salud son numerosos y, por lo general, el tiempo destinado a la asignatura dentro de las mallas es limitado. En un estudio realizado en Estados Unidos(21), en que se revisaron los contenidos de los cursos de ética de 87 escuelas de medicina se concluyó que existe gran variación entre ellas. De las 37 áreas temáticas arrojadas por el estudio sólo el consentimiento informado fue un tema incluido en la mayoría de los programas
(87\%). De los temas restantes, sólo seis fueron mencionados por al menos la mitad de las escuelas que contestaron la encuesta. En el ámbito odontológico la literatura es más escasa al respecto; sin embargo, varios autores $(4,12,14,19,20,22,23)$ ofrecen una guía de los tópicos que deberían ser abordados en el programa de ética en la carrera de odontología bajo diferentes propuestas de sistematización. Estos contenidos se resumen a continuación:

\begin{tabular}{|l|l|}
\hline - Consentimiento informado & $\bullet$ Necesidades de los pacientes \\
\hline - Rechazo de planes de tratamiento & $\bullet$ Conflictos entre profesionales \\
\hline - Relación profesional-paciente & $\bullet$ Negligencia médica \\
\hline - Relación profesional-paciente-familia & $\bullet$ Toma de decisiones \\
\hline - Relación clínica veraz y buena comunicación & $\bullet$ Pacientes de carácter difícil \\
\hline - Confidencialidad y buena práctica clínica & $\bullet$ Competencias y habilidades clínicas de los estudiantes \\
\hline - Investigación médica y odontológica & $\bullet$ Temas culturales \\
\hline - Pacientes vulnerables: Niños, pacientes con desórdenes mentales e incapacidad & $\bullet$ Incumplimiento de confidencialidad \\
\hline o con problemas sistémicos. & $\bullet$ Información no relevada por el paciente \\
\hline - Asignación de recursos y ética en la administración & $\bullet$ Temas legales \\
\hline - Derechos, deberes y responsabilidades de profesionales y pacientes & \\
\hline
\end{tabular}

\section{Estrategias para la enseñanza de la ética}

Así como la visión y manera de incorporar la ética en el currículo ha ido evolucionando, también lo han hecho las tendencias y estrategias utilizadas para llevar a cabo su enseñanza. Esta evolución se suma a la que se está produciendo en el ámbito educacional en general, en el cual el proceso centrado en el estudiante, a través de estrategias más participativas y vivenciales, logra un aprendizaje de mejor calidad y más duradero.

La principal metodología usada en los nuevos currículos para enseñar ética es el aprendizaje basado en problemas (PBL). Esta técnica favorece el desarrollo de habilidades y actitudes con relación a temas éticos, estimula el trabajo en equipo y la capacidad de escuchar otras opiniones. Además, facilita el razonamiento ético y la toma de decisiones por parte del estudiante $(4,5,14,24)$. Los casos del PBL son derivados de situaciones o dilemas que ocurren en el diario ejercicio de la profesión odontológica. Esto estimula al educando a reconocer problemas, manejar la duda e incertidumbre ante ciertas situaciones, argumentar y fundamentar sus ideas e intercambiar opiniones que ayuden a clarificar su propio punto de vista(12).

Otra metodología fuertemente promovida en las escuelas de medicina es el aprendizaje basado en casos.
Perkins sugirió que la mejor forma de enseñar ética médica es a través de pacientes reales, método sugerido por la mayoría de los educadores en el área de la odontología como una forma de capturar la atención de los estudiantes y hacer la instrucción de la ética clínicamente relevante y motivadora $(11,13)$.

Un programa desarrollado e implementado por el profesor Joseph Healey en las escuelas de Odontología y Medicina de la Universidad de Connecticut, desde 1975, recibe el nombre de "Aspectos Éticos y Legales de la Medicina y la Odontología (LEA)". Consiste en 56 casos actuales e hipotéticos creados por el autor, desde los cuales los estudiantes extraen los principios éticos y morales. El curso desarrolla en los estudiantes la capacidad de comprender el carácter ético y legal de las decisiones que ellos toman como profesionales de la salud y los hace ganar experiencia en la aplicación de principios. Este programa tiene tres objetivos principales, llevados a cabo por medio de discusión con un facilitador, panel de expertos, plenarios y conferencias(23):

- Desarrollar sensibilidad ante la presencia de asuntos éticos y legales en el área de la salud, para que estos temas puedan ser identificados a medida que surjan en la práctica clínica. 
- Capacitar a los estudiantes para que aprendan a describir y presentar temas éticos y legales para así llegar a acuerdos constructivos.

- Capacitar a los estudiantes para examinar sus propios valores y descubrir como éstos se relacionan con sus responsabilidades profesionales.

Otra estrategia es el llamado "Test dental de sensibilidad ética" (DEST), que consiste en ocho videos creados para la enseńanza y evaluación ética. Los temas y situaciones presentados se derivan de reportes sobre problemas éticos que frecuentemente ocurren en odontología, con representaciones acuciosamente chequeadas por dentistas y otros especialistas. Su validez y confiabilidad han sido reportadas en varios estudios, a través de los cuales se ha podido concluir que:

- La sensibilidad ética puede ser evaluada confiablemente.

- Los estudiantes presentan gran variación en su habilidad para reconocer problemas éticos de su profesión y esta habilidad es distinta de las capacidades de razonamiento moral. En otras palabras, los estudiantes pueden ser capaces de interpretar la dimensión ética de una situación (sensibilidad ética), pero incapaces de percibir una solución moral.

- La sensibilidad ética puede ser desarrollada a través de la instrucción.

Helen Sharp y colaboradores proponen que los contenidos tengan directa relación con las inquietudes de los estudiantes en este ámbito. Para esto diseñaron una estrategia en la cual cada alumno debe registrar en una bitácora las situaciones o dilemas éticos que encuentra en su quehacer clínico. Posteriormente se realiza un análisis de estas recopilaciones generando así instancias de discusión que favorecen el aprendizaje de la ética(22).
En la literatura también se describen otras metodologías utilizadas con menos frecuencia en la enseñanza de la ética: estudio de casos, discusión en sesiones clínicas, mesas redondas, paneles, lectura dirigida, entre otros(4,11-13).

\section{Conclusiones}

Entender que la dimensión ética de la odontología es un elemento esencial en el óptimo desempeńo de los futuros profesionales es, sin duda, la clave para posibilitar la incorporación de la ética como una instancia formal dentro del currículo.

Es necesario reconocer, además, que la formación universitaria de pregrado es el momento propicio para abordar estos temas, abandonando la creencia de que la formación valórica es inalterable y, por tanto, no es función de la universidad participar en su desarrollo.

La ética debe ser enseñada desde un punto de vista práctico y con relación a situaciones a las cuales los estudiantes se ven enfrentados en su vida profesional. Por lo tanto, las estrategias deben obedecer a estas demandas y favorecer cambios conductuales que los lleven a una correcta toma de decisiones dentro de un marco sociocultural real. Parece ser, según lo observado en los nuevos currículos, que las metodologías centradas en el estudiante (como el aprendizaje basado en problemas) proporcionan la mejor forma de lograr estos objetivos.

La educación ética dentro de los currículos de las profesiones de la salud no deja de ser compleja y difícil de implementar. Esto se debe, entre otras cosas, al carácter multifactorial e interprofesional de ciertos dilemas éticos, la falta de profesionales capacitados, poco interés por parte de los educadores y la resistencia de instancias superiores a realizar modificaciones curriculares importantes.

\section{Referencias}

1. Guerra R. Bioética en la malla curricular de Odontología. Acta Bioethica 2006; 12(1): 49-54.

2. Aires C, Neves F, Rosalen P, Marcondes F. Teaching of bioethics in dental graduate programs in Brazil, Bras Oral Res 2006; 20(4): 285-289.

3. Asai A, Minaka K, Tsuguya F, Masano T. Postgraduate education in medical ethics in Japan. Med Educ 1998; 32: 100104.

4. De Amorín A, Ferreira E, Freitas R. O lugar da Bioética nos currículos de formacao médica. Rev Bras Educ Med 2006; 30(2): 56-65.

5. Siqueira J. O ensino da ética no curso de medicina: a experiencia da Universidade estadual de Londrina (UEL). Bioética 2002;10(1): 85-95. 
Ética en el currículo de las carreras de odontología - Carlos Zaror Sánchez y Carolina Vergara González

6. Miles SH, Lane LW, Bickel J, et al. Medical Ethics Education: coming of age. Acad Med 1989; 64: 705-714.

7. Buendía A, Álvarez de la Cadena C. Nivel de conocimiento de la bioética en carreras de odontología de dos universidades de América Latina. Acta Bioethica 2006; 12(1): 41-47.

8. Odom JG, Beemsterboer PL, Pate TD, Haden NK. Revisiting the status of dental ethics instruction. J Dent Educ 2000; 64(11): 772-783.

9. Bertolami CH. Why our ethics curricula don't work. J Dent Educ 2004; 68(4): 414-425.

10. Ozar D. Formal instruction in dental professional ethics. J Dent Educ 1985; 49: 696-701.

11. Fox E, Arnold, R, Brody B. Medical ethics education. Past, Present, Future. Acad Med 1995; 70: 761-769.

12. Goldie J. Review of ethics curricula in undergraduate medical education. Med Educ 2000; 34: 108-119.

13. Berk N. Teaching ethics in dental schools: Trends, techniques, and targets. J Dent Educ 2001; 65(8): 744-750.

14. Goldie J, Schwartz L, McConnachie A, Morrison J. Impact of a new course on students' potential behavior on encountering ethical dilemmas. Med Educ 2001; 35: 295-302.

15. Dávila R. É possível ensinar ética médica em um curso formal curricular? Bioética 2002; 10(1): 115-126.

16. Nash D. On ethics in the profession of dentistry and dental education. Eur J Dent Educ 2007; 11: 64-74.

17. Larios H, Paredes R, Ortigosa J. Ética en la formación médica. Disponible en http://www.facmed.unam.mx/eventos/ seam $2 \mathrm{k} 1 / 2004 /$ ponencia_feb_2k4.htm

18. Cardozo C, Rodríguez E, Lolas F, Quezada A. Ética y Odontología. Una Introducción. Santiago de Chile: CIEB, Universidad de Chile; 2006: 117-128.

19. Bridgman A, Collier A, Cunningham J, et al. Teaching and assessing ethics and law in the dental curriculum. Br Dent J. 1999; 187(4): 217-219.

20. Bebeau M. Can Ethics be Taught? A look at the evidence. Disponible en: https://www.dentalethics.org/index.shtml

21. Dubois JM, Burkemper J. Ethics education in U.S. medical schools: a study of syllabi. Acad Med 2002; 77: 432-437.

22. Sharp H, Kuthhy R, Heller K. Ethical dilemmas reported by fourth-year dental student. J Dent Educ. 2005; 69(10): 1116-1122.

23. Blechner, B, Hager $\mathrm{CH}$, William N. The Jay Haley Technique: Teaching law and ethics to medical and dental students. Am j Law Med 1994; 20(4): 439-455.

24. Parker M. Autonomy, problem based-learning, and the teaching of medical ethics. J Med Ethics 1995; 21: 305-310.

Recibido: 4 de junio de 2008

Aceptado: 18 de julio de 2008 